\title{
Comparing Performance of Text Pre-processing Methods for Predicting a Binary Position by LASSO
}

\author{
Experiment with Textual Data of European Union Public Consultation
}

\author{
Nada Mimouni \\ Governance Analytics, University Paris-Dauphine \\ PSL Research University \\ Paris, France \\ nada.mimouni@dauphine.fr
}

\author{
Timothy Yu-Cheong Yeung \\ Governance Analytics, University Paris-Dauphine \\ PSL Research University \\ Paris, France \\ yu-cheong.yeung@dauphine.fr
}

\begin{abstract}
This work aims at comparing different methods of preparing textual inputs for LASSO logistic regression to predict a binary position with the textual data extracted from a public consultation of the European Commission. Texts are pre-processed and then input into LASSO to explain the stakeholder's position, and the mean squared errors are computed to compare different methods. In short, we do not find a clearcut winner. On average, tf-idf performs better than counts of distinct terms, and deleting terms that appear only once reduces the prediction errors.
\end{abstract}

\section{CCS CONCEPTS}

- Computing methodologies $\rightarrow$ Information extraction; Classification and regression trees;

\section{KEYWORDS}

Text analysis, LASSO logistic regression, European Union, Public consultations

\section{INTRODUCTION}

Textual analysis has recently become popular in social sciences, thanks to the advances of computer sciences. However, the quality of the analysis depends on the preparation of the inputs. "Garbage in, Garbage out" often happens if the practitioners are unaware of the importance of the preprocessing of the texts. This paper aims at comparing different methods of feature selection for predicting a binary dependent variable. We contrast methods in three dimensions. First, we contrast lemmatization and stemming. Second, we prepare a sample that includes all terms and a sub-sample that excludes the terms that appear only once in the corpus. Finally, we either use the counts of distinct terms as inputs or compute the tf-idf values of each term. Feature selection is done by LASSO (least absolute shrinkage and selection operator) logistic regression that is a form of penalized linear models [16]. Different methods are then evaluated by the size of mean squared errors.

Permission to make digital or hard copies of part or all of this work for personal or classroom use is granted without fee provided that copies are not made or distributed for profit or commercial advantage and that copies bear this notice and the full citation on the first page. Copyrights for third-party components of this work must be honored. For all other uses, contact the owner/author(s)

The Second Women in Data Science (WinDS) Workshop, co-located with The Web Conference 2018, April 24, 2018, Lyon, France.

(c) 2018 Copyright held by the owner/author(s).

https://doi.org/10.18122/winds/1/boisestate
The data are collected from a public consultation launched by the European Commission on roaming wholesale market. Each response to a question in the consultation is considered as a document. We select four questions which contain a check-box and an open text-box. The information given in the check-box helps us construct the binary position of the document on a certain matter. The open text-box allows us to extract additional textual information that supports the document's position. The LASSO regression will pick those terms which are more able in explaining the documents' positions and give a weight to each chosen term. The expected predicted values are then compared with the actual positions, and the most accurate method should give the lowest mean squared errors (MSE).

Public consultations by the European Commission are designed for the stakeholders and the public to participate in the decisionmaking process. Their positions and any supporting arguments could be taken by the Commission and written into the directive issued to all member states. It is arguably an important channel for stakeholders to exert influence at the European level and thus most of them take it seriously. Besides, public consultations well-suit our purpose because they are systematically designed, vary in topics, and contain useful information for research in other disciplines.

Our work's contributions are at least threefold. First, the prediction helps us complete the sample by filling in missing values as some stakeholders for some reason did not give a clear answer with the check-box. Second, the obtained coefficients of terms help us construct a bag of related terms for each position along a dimension of a topic or policy. That helps us classify any related documents along the same dimension. Third, this work sheds light on the search of the best method of text pre-processing in text classification.

\section{LITERATURE REVIEW}

Feature selection methods have been widely researched in different contexts [8]. Text classification is often the focus, which means assigning classes to documents according to their content. The development of support vector machines even calls for the avoidance of lemmatization and stemming $[9,12]$, but the debate is still lively . Removing stop words is generally accepted as helpful but both lemmatization and stemming worsen the performance [17]. On the other hand, choosing appropriate preprocessing tasks is shown to improve significantly the classification accuracy [18].

Meanwhile, textual analysis is becoming more and more popular in social sciences $[3,15]$. It has been widely applied in finance to 
understand the amount, the tone and the transparency of the disclosure [10]. An index of media slant is constructed to measure the similarity of the language used by the newspapers and politicians [6]. Words used in newspapers are taken to measure policy uncertainty [2]. Movie reviews are used to predict revenues by LASSO regression [7].

\subsection{Automatic Text Processing}

2.1.1 Text mining. The web has become the main channel to communicate and to share information among people. A huge amount of data is thus produced every day and published on the web. A wide variety of approaches and methods are developed to automatically analyze this data and extract hidden useful knowledge. In particular, text mining is a branch of data mining focusing on the analysis of voluminous and unstructured text data. It derives high-quality information from text through the process of structuring the input, extracting motifs from the structured data, then evaluating and interpreting the output.

The first step is mainly based on the use of natural language processing (NLP) techniques, such as syntactic parsing, part of speech tagging (POS). The second step involves algorithms for text classification (naive Bayes, nearest neighbor, decision tree), text clustering (hierarchical algorithms, k-means, probabilistic and topic models) or information extraction (named entity recognition, relation extraction, etc.)[1]. In our research, we are using the Penalized Logistic Regression (LASSO) as a classifier for binary text categorization which we apply to the prediction of authors positions.

2.1.2 Text Preprocessing. Processing large collections of documents is a complex and challenging task for text mining algorithms. The first step of all is text preprocessing where essentially the text is turned into data for further analysis. The output from this stage has big impact on the analysis stage as shown by the authors in [18] for the specific field of text classification. Natural language processing, statistical and analytical techniques are the main actors for this step. The tasks of preprocessing are mainly tokenization, filtering, stemming and lemmatization, which prepare the input to generate a vector space representation.

\section{THE DATA}

The policy-making of the European Union is chosen as the target domain. In a regular manner, the European Commission involves stakeholders in the legislation process via open public consultations. A consultation is presented as a list of questions each attached with a list of pre-defined answers (check-boxes) and a free text area where users can explain their choices. All consultations from the Commission are open and are gathered in one list accessible in their web site ${ }^{1}$.

In this study, we choose the Public consultation on the review of national wholesale roaming markets, fair use policy and the sustainability mechanism referred to in the Roaming Regulation 531/2012 as amended by Regulation 2015/2120. In total, we collected 43 responses to the consultation from the stakeholders, who are mainly companies and trade unions, and the proposed directive (to the European Parliament) of the Commission. We manually studied the proposed

${ }^{1}$ Consultations of the European Commission : https://ec.europa.eu/info/consultations en directive that was drafted after the consultation in order to extract the final decisions with respect to the questions of the consultation. We finally pick 4 questions from the consultation for which we can identify the exact final position of the Commission. Next, we extracted the answers from the check-boxes manually to identify the positions of the stakeholders on these 4 questions, and from them we construct a binary variable that corresponds to whether the stakeholder's position is aligned with the Commission's (value 1) or not (value O). Information given in the open-boxes is then extracted by automated techniques.

\section{METHODS TO PREPARE THE INPUTS}

The collected data consists of 43 responses coming from 43 different stakeholders to the consultation on roaming markets. From the manual analysis, we selected 4 questions and thus in total the dataset contains 172 documents. For technical aspects of this study we used the python libraries scipy and nltk for NLP, gensim to generate bi-grams and sklearn for features extraction (to create the vector space model).

\subsection{Cleaning, Filtering and Tokenization}

Cleaning consists of removing all special characters from the original text, such as delimiters from html or urls, etc. Blank spaces and line breaks are also removed. In order to avoid any problems related to the encoding of characters, we decoded the text to 'utf8' as the most standard text encoding format and fixed unicode problems.

For filtering, we used a pre-compiled stop-words list for English. We also defined a new list of domain-specific terms that could be removed. This is a good practice to avoid those frequent terms but not very important for defining the semantics of a given domain. The stream of text is then broken into pieces to generate a list of tokens. We generated bi-grams (sequences of two words that appear more then a fixed threshold) to better capture the semantics of text classes.

\subsection{Lemmatization and Stemming}

Both lemmatization and stemming aim at reducing inflectional forms of a word to a common base form [13]. Stemming is used to reduce words to their root by deleting prefixes and suffixes. In this study, we used the Porter's stemming algorithm [14]. Lemmatization uses vocabulary and morphological analysis of words to return the base or the dictionary form of a word. To experiment the effect of using one or the other technique, we used combinations with terms frequency and POS-tags of words to generate different test corpora.

\section{LASSO REGRESSION AND RESULT COMPARISON}

LASSO logistic regression is the recommended regression method for text classification[5]. LASSO regression stands out among other text classification methods especially in social sciences because of its simple implementation and interpretation. It solves the problem of dimensionality that complicates textual analysis because inputs (tokens) are too numerous compared to a standard size of sample. It penalizes those variables with large coefficients by adding a penalty term in the process of maximizing the log-likelihood. With 
Table 1: Input dataset example - the question Q20

\begin{tabular}{ll}
\hline Question text & $\begin{array}{l}\text { Do you consider that the functioning of the national wholesale roaming markets absent regulation } \\
\text { would be capable of delivering RLAH at the retail level in accordance with the domestic charging model? }\end{array}$ \\
$\begin{array}{ll}\text { Check-box options } & \text { "Yes"; "No"; "It depends on the Member State"; "Don't know". } \\
\text { Free text-box } & \text { Please explain your response and provide examples: }\end{array}$ \\
\hline
\end{tabular}

a proper weight (often mentioned as $\lambda$ or $\lambda_{1}$ in the literature) chosen, LASSO assigns zeros to some coefficients and helps reducing the dimensions significantly. Precisely, we write the penalized linear model as follows:

$$
\min \left\{l(\alpha, \beta)+n \lambda \sum_{j=1}^{p} \omega_{j}\left|\beta_{j}\right|\right\}
$$

where $l(\alpha, \beta)$ is the negative log likelihood function in the binomial logistic regression, $n$ is the total number of documents, and $\lambda$ is the weight given to each penalty term, which is $\omega_{j}\left|\beta_{j}\right|$ for term $j=1,2 \ldots, p$. We standardized each coefficient by the standard deviation of that covariate. In effect, the standardization will give a larger weights to rare terms and is recommended in the literature [11].

Lemmatization and stemming prepare the inputs differently, and thus LASSO will give different outputs. For example, A and B wrote "regulation" and "regulatory" respectively. Stemming groups two words together, and therefore "regul" as an input appears two times. But lemmatization does not consider them as equal and each word is a distinct input that appears only once. When predicting a position that is associated with regulation in general, we may question whether lemmatization really values the concept sufficiently. Moreover, we employ POS-tag to keep only nouns in the sample before stemming. To further reduce the size of inputs, we consider also a subsample that excludes those terms that appear only once. Apart from reducing the dimensionality, we consider both counts of distinct terms and tf-idf values. In effect, deleting those words that appear only once does not make a difference when working with counts because LASSO will consider their unimportance and assign zeros to them. Therefore, we compare in total 9 different methods (see Table 2).

As mentioned above, each selected question has a check-box for the contributors to precisely state their position and is followed by an open-box for any additional comments on the issue. We can thus identify where the stakeholders actually stands, either aligned with the Commission or not, which is the dependent variable of our LASSO regression, while we capture the textual inputs in the openbox and use them as the explanatory variables in the regression. As our objective is to predict a contributor's position, the best set of inputs should give the smallest errors. We thus compute the mean squared errors of the 9 types of inputs for the 4 questions. The mean squared errors are computed as follows:

$$
M S E=\frac{1}{n} \sum_{i=1}^{n}\left(y_{i}-\hat{y}_{i}\right)^{2}
$$

The actual value $y_{i}$ is either 1 (aligned with the Commission ex-post) and 0 (not aligned). The predicted value $\hat{y}_{i}$ is the prediction of the LASSO regression, which ranges from 0 to 1 . A smaller MSE value says that the prediction is more accurate. Therefore, we can compare the values and may be able to conclude if one feature selection method is superior than others.

Besides, LASSO is usually done with frequencies as the inputs. The tf-idf is an alternative, which increases a term's weight proportionally to the number of times it appears in the document, but offset by the frequency of it in the corpus. Values after the transformation are smaller than 1, and LASSO is unable to work with too small values. We therefore adjust the values by multiplying them by 10 before inputing in the LASSO regression. Since we standardized the coefficients of LASSO and transformed the inputs into tf-idf, rare terms are weighted up twice. Our results will also tell if it overweights rare terms and worsens the prediction.

The choice of $\lambda$ is subject to debate. Some recommend to select the value that minimizes the average error, while some others propose to use a high-dimension-adjusted Akaike Information Criteria [4]. Due to the relatively low dimensions of our corpus (from 73 to 689), we cannot afford losing too many information. Therefore, we simply picked the smallest value of $\lambda$ that gives a number of estimates which is smaller than the number of all unique terms. Consequently, $\lambda$ ranges from 1 to 4 . The number of non-zero coefficients ranges from 5 to 19 . Table 2 reports the $\lambda$ chosen and the number of non-zero coefficients of the LASSO regression.

Table 3 reports the comparison of results of the 9 methods. We highlight the lowest MSE value for each question. In short, we do not find an absolutely superior method of preparing the inputs for LASSO. It shows that the research question indeed does not have an obvious answer and needs further investigation. Tf-idf (min frequency $=1$ ) beats counts 7 out of 12 times and tf-idf (min frequency $=2$ ) beats counts 9 out of 12 times. Tf-idf $(\min$ frequency $=$ 2) performs better than tf-idf ( $\min$ frequency= 1) 9 out of 12 times. If we only consider counts, stemming with POS-tag beats others 3 out of 4 times. If we only consider tf-idf (min frequency $=2$ ), stemming with POS-tag performs better than others 3 out of 4 times. On average, stemming plus POS-tag with tf-idf (min frequency $=2$ ) gives the lowest average MSE. It is also the method that gives the highest number of non-zero coefficients. It suggests that LASSO works better if the inputs have been significantly trimmed.

Stemming seems to be a superior choice. Lemmatization may allow too many forms of the same concept to appear separately, that in effect diminishes their importance relative to some terms which do not have many different forms. For example, the root "regul" is in certain context more important than "market", but the former could be expressed in "regulation", "regulatory", etc. Tf-idf performs on average better than counts because of its advantage to value more the rare terms. Besides, we find no evidence that $\mathrm{tf}$-idf transformation should not be employed together with standardizing coefficients in LASSO regression. Weighting up rare terms twice 
Table 2: Summary of $\lambda$ and Non-zero Coefficients of 9 Methods

\begin{tabular}{|c|c|c|c|c|}
\hline & Q20 & Q25 & Q26 & Q27 \\
\hline \multicolumn{5}{|c|}{ Method $1:$ Lemma+Counts min freq $=1$} \\
\hline$\lambda$ & 2 & 3 & 2 & 2 \\
\hline Non-zero coefficients & 9 & 6 & 7 & 5 \\
\hline \multicolumn{5}{|c|}{ Method 2: Lemma+tfidf min freq $=1$} \\
\hline 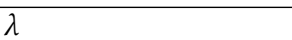 & 2 & 2 & 2 & 3 \\
\hline Non-zero coefficients & 13 & 9 & 8 & 8 \\
\hline \multicolumn{5}{|c|}{ Method $3:$ Lemma+tfidf $\min$ freq $=2$} \\
\hline 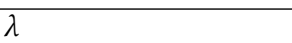 & 3 & 3 & 3 & 3 \\
\hline Non-zero coefficients & 10 & 12 & 13 & 10 \\
\hline \multicolumn{5}{|c|}{ Method $4:$ Stem + Counts min freq $=1$} \\
\hline$\lambda$ & 2 & 2 & 2 & 2 \\
\hline Non-zero coefficients & 10 & 9 & 8 & 6 \\
\hline \multicolumn{5}{|c|}{ Method $5:$ Stem + tfidf $\min$ freq $=1$} \\
\hline$\lambda$ & 2 & 3 & 2 & 3 \\
\hline Non-zero coefficients & 6 & 9 & 11 & 8 \\
\hline \multicolumn{5}{|c|}{ Method $6:$ Stem + tfidf $\min$ freq $=2$} \\
\hline$\lambda$ & 2 & 3 & 2 & 3 \\
\hline Non-zero coefficients & 7 & 10 & 11 & 11 \\
\hline \multicolumn{5}{|c|}{ Method $7:$ Stem+POStag+Counts min freq =1 } \\
\hline$\lambda$ & 2 & 2 & 1 & 2 \\
\hline Non-zero coefficients & 10 & 8 & 11 & 5 \\
\hline \multicolumn{5}{|c|}{ Method $8:$ Stem+POStag+tfidf min freq =1 } \\
\hline$\lambda$ & 3 & 3 & 2 & 4 \\
\hline Non-zero coefficients & 10 & 12 & 11 & 5 \\
\hline \multicolumn{5}{|c|}{ Method $9:$ Stem+POStag+tfidf min freq =2 } \\
\hline$\lambda$ & 3 & 2 & 3 & 3 \\
\hline Non-zero coefficients & 19 & 13 & 16 & 13 \\
\hline Sample Size & 34 & 41 & 39 & 35 \\
\hline
\end{tabular}

Table 3: Result Comparison

\begin{tabular}{lcccc}
\hline \hline MSE & Q20 & Q25 & Q26 & Q27 \\
\hline Lemmatization & & & & \\
\hline Method 1 & 0.16900 & 0.16279 & 0.12581 & 0.19358 \\
\hline Method 2 & $\mathbf{0 . 1 6 3 4 2}$ & 0.26995 & 0.10801 & 0.18665 \\
\hline Method 3 & 0.19548 & 0.12005 & 0.09230 & 0.16459 \\
\hline Stemming & & & & \\
\hline Method 4 & 0.16394 & 0.26207 & 0.11248 & 0.20035 \\
\hline Method 5 & 0.20257 & 0.13818 & 0.09744 & 0.19463 \\
\hline Method 6 & 0.19925 & 0.12412 & 0.08930 & 0.17784 \\
\hline Stemming+POStag & & & & \\
\hline Method 7 & 0.18635 & 0.11629 & $\mathbf{0 . 0 8 3 1 5}$ & 0.19324 \\
\hline Method 8 & 0.19893 & 0.12005 & 0.09060 & 0.19012 \\
\hline Method 9 & 0.17489 & $\mathbf{0 . 0 8 1 1 5}$ & 0.15997 & $\mathbf{0 . 1 4 5 6 0}$ \\
\hline \hline
\end{tabular}

performs better compared to the baseline result using counts. POStag that keeps only nouns tends to work well in our context.

\section{CONCLUSION}

This work aims at comparing different ways of pre-processing of texts for predicting a binary position by LASSO logistic regression.
We collect textual data from a public consultation launched by the European Commission and manually identify alignments and misalignments between stakeholders and the Commission, which are explained by the textual inputs given in the open-box. Although we do not find a clearcut winner, tf-idf on average performs better than counts, and deleting terms that appear only once tends to reduce the prediction errors. In short, stemming plus POS-tag with tf-idf and minimum term frequency equal to 2 generates the lowest average mean squared errors.

\section{REFERENCES}

[1] Mehdi Allahyari, Seyed Amin Pouriyeh, Mehdi Assefi, Saied Safaei, Elizabeth D. Trippe, Juan B. Gutierrez, and Krys Kochut. 2017. A Brief Survey of Text Mining: Classification, Clustering and Extraction Techniques. In Proceedings of the KDD bigdas. ACM Press, Halifax, Canada, 13.

[2] Scott R Baker, Nicholas Bloom, and Steven J Davis. 2016. Measuring economic policy uncertainty. The Quarterly fournal of Economics 131, 4 (2016), 1593-1636.

[3] Norman Fairclough. 2003. Analysing discourse: Textual analysis for social research. Psychology Press.

[4] Cheryl J Flynn, Clifford M Hurvich, and Jeffrey S Simonoff. 2013. Efficiency for regularization parameter selection in penalized likelihood estimation of misspecified models. 7. Amer. Statist. Assoc. 108, 503 (2013), 1031-1043.

[5] Matthew Gentzkow, Bryan T Kelly, and Matt Taddy. 2017. Text as data. Technical Report. National Bureau of Economic Research.

[6] Matthew Gentzkow and Jesse M Shapiro. 2010. What drives media slant? Evidence from US daily newspapers. Econometrica 78, 1 (2010), 35-71.

[7] Mahesh Joshi, Dipanjan Das, Kevin Gimpel, and Noah A Smith. 2010. Movie reviews and revenues: An experiment in text regression. In Human Language Technologies: The 2010 Annual Conference of the North American Chapter of the Association for Computational Linguistics. 293-296.

[8] Alan Jović, Karla Brkić, and Nikola Bogunović. 2015. A review of feature selection methods with applications. In Information and Communication Technology, Electronics and Microelectronics (MIPRO), 2015 38th International Convention on. IEEE, 1200-1205.

[9] Edda Leopold and Jörg Kindermann. 2002. Text categorization with support vector machines. How to represent texts in input space? Machine Learning 46, 1-3 (2002), 423-444.

[10] Feng Li. 2010. Textual analysis of corporate disclosures: A survey of the literature. Journal of accounting literature 29 (2010), 143.

[11] Christopher D Manning, Prabhakar Raghavan, and Hinrich Schütze. 2008. Introduction to Information Retrieval. Cambridge University Press.

[12] José Ramon Méndez, Eva Lorenzo Iglesias, Florentino Fdez-Riverola, Fernando Díaz, and Juan M Corchado. 2005. Tokenising, stemming and stopword removal on anti-spam filtering domain. In Conference of the Spanish Association for Artificial Intelligence. Springer, 449-458.

[13] Christopher D. Mnning, Prabhakar Raghavan, and Hinrich Schutze. 2008. Introduction to Information Retrieval. Cambridge University Press, Chapter Stemming and lemmatization. https://nlp.stanford.edu/IR-book/

[14] Martin F. Porter. 1980. An algorithm for suffix stripping. Program 14, 3 (1980), 130-137.

[15] Steve Stemler. 2001. An overview of content analysis. Practical assessment, research \& evaluation 7, 17 (2001), 137-146.

[16] Robert Tibshirani. 1996. Regression shrinkage and selection via the lasso. fournal of the Royal Statistical Society. Series B (Methodological) (1996), 267-288.

[17] Michal Toman, Roman Tesar, and Karel Jezek. 2006. Influence of word normalization on text classification. Proceedings of InSciT 4 (2006), 354-358.

[18] Alper Kursat Uysal and Serkan Gunal. 2014. The impact of preprocessing on text classification. Information Processing \& Management 50, 1 (2014), 104-112. 